Nouvelles perspectives en sciences sociales

Revue internationale de systémique complexe et d'études relationnelles

\title{
Le lieu agricole périurbain : un analyseur de la complexité des constructions territoriales entre actions politiques, débats publics et pratiques spatiales Étude de cas dans le Lunellois (Languedoc)
}

\section{Camille Clément}

Volume 10, numéro 1, novembre 2014

Sur le thème du territoire

URI : https://id.erudit.org/iderudit/1028436ar

DOI : https://doi.org/10.7202/1028436ar

Aller au sommaire du numéro

Éditeur(s)

Prise de parole

ISSN

1712-8307 (imprimé)

1918-7475 (numérique)

Découvrir la revue

Citer cet article

Clément, C. (2014). Le lieu agricole périurbain : un analyseur de la complexité des constructions territoriales entre actions politiques, débats publics et pratiques spatiales : étude de cas dans le Lunellois (Languedoc). Nouvelles perspectives en sciences sociales, 10(1), 27-57. https://doi.org/10.7202/1028436ar
Résumé de l'article

Cet article a pour objectif d'éclairer la complexité des constructions et appropriations territoriales à partir de l'étude croisée des actions politiques, débats publics et pratiques spatiales de la communauté de communes du Pays de Lunel (Languedoc). En cours de périurbanisation, ce territoire mise sur son ancrage agricole et rural pour se différencier des agglomérations en expansion de Montpellier et de Nîmes. C'est donc par la thématique agricole qu'une étude qualitative du SCOT (Schéma de Cohérence Territoriale), d’un projet de circuits courts et d'un pôle oenotouristique (actions politiques) ont été étudiés afin de saisir cinq débats publics (étude de la presse régionale et intercommunautaire) qui sont directement en lien avec les choix politiques réalisés par cette instance territoriale. Au final, l'étude montre que ces actions politiques et débats publics doivent être mis en relation avec les pratiques spatiales observées dans le territoire. Ce n'est qu'à l'échelle du lieu agricole périurbain que ces trois notions s'alimentent mutuellement afin de montrer la complexité des appropriations territoriales, appropriations par le politique (actions politiques et débats publics) et appropriations par la pratique. 


\section{Le lieu agricole périurbain : un analyseur de la complexité des constructions territoriales entre actions politiques, débats publics et pratiques spatiales}

\section{Étude de cas dans le Lunellois (Languedoc)}

\section{Camille Clément}

UMR Innovation (Inra-Cirad-SupAgro), Montpellier

ISARA Lyon - équipe ASTER, Lyon

\section{Introduction}

T a notion de territoire n'est pas consensuelle en géographie. $\triangle$ Parfois dénoncé comme vide de sens tant il en contient ${ }^{1}$, le territoire n'en demeure pas moins une notion structurante pour la discipline. Certaines contributions résument ces divergences et analysent les différentes définitions avancées par les géographes français ${ }^{2}$. Nombre d'entre elles reprennent la notion éthologique du terme en parlant d'appropriation, au départ animale, à présent sociale et politique. Cette appropriation spatiale serait multiple et recouvrirait ou croiserait les dimensions «politiques, culturelles

\footnotetext{
$1 \quad$ Benoît Anthaume et Frédéric Giraut, Le territoire est mort, vive les territoires! Une (re)fabrication au nom du développement, Paris, IRD Éditions, 2005.

2 Frédéric Giraut, "Conceptualiser le territoire», Historiens et géographes, n ${ }^{\circ}$ 403, 2008, p. 57-68; Jacques Lévy et Michel Lussault, Dictionnaire de la géographie et de l'espace des sociétés, Paris, Belin, 2003.
} 
et économiques ${ }^{3}$ » ou "économique, idéologique et politique $e^{4}$ » qui fondent le territoire. Nous pourrions alors résumer les débats à propos de la notion à un jeu de "proportion» entre ces dimensions. Tantôt dominé par la dimension politique, le territoire deviendrait idéologique ou support spatial du pouvoir ${ }^{5}$. Cette approche du territoire rejoint les travaux de Michel Foucault sur le territoire de la "discipline», ses dispositifs spatiaux coercitifs ${ }^{6}$. Dominé par le culturel, le territoire se définit par sa dimension socio-culturelle qui désigne une appropriation spatiale communautaire ${ }^{7}$. La question est alors de savoir comment se jouent l'identité communautaire et son fonctionnement plus ou moins intégrateur. Enfin, dominé par l'économique, il désigne le champ du développement territorial et de la ressource territoriale, facteurs de croissance économique ${ }^{8}$.

Pour étudier un territoire, le chercheur va donc commencer par l'objectiver. Autrement dit, il va définir ses objectifs et choisir la ou les dimensions par lesquelles il saisira le territoire qui est son objet d'étude. Mais ce territoire a également une existence en soi qui résulte de constructions multiples de la part des acteurs, qu'ils soient élus, techniciens dans des établissement public de coopération intercommunale (EPCI), responsables ou adhérents d'associations d'animation du territoire ou simples habitants ${ }^{9}$. Il convient alors de saisir ces constructions territoriales dans leur complexité. C'est ce que cette étude de cas, centrée sur le territoire du Lunellois, tente de faire 1) en partant d'actions

3 Frédéric Giraut, op. cit.

4 Guy Di Méo, Géographie sociale et territoires, Paris, Nathan Université, 2001.

5 Michel Lussault, L'Homme spatial. La construction sociale de l'espace humain, Paris, Seuil, 2007.

6 Michel Foucault : Naissance de la biopolitique. Cours au collège de France, Paris, Presses universitaires de France, 2004; Surveiller et puni. Naissance de la prison, Paris, Gallimard, 1975.

7 Bernard Debarbieux, «Le lieu, le territoire et trois figures de rhétorique», Espace géographique, vol. 24, $\mathrm{n}^{\circ}$ 2, 1995, p. 97-112; Philippe GervaisLambony, Territoires citadins : 4 villes africaines, Paris, Belin, 2003.

8 Hervé Gumuchian et Bernard Pecqueur, La ressource territoriale, Paris, Economica, 2007.

9 Hervé Gumuchian et al., Les acteurs, ces oubliés du territoire, Paris, Economica, 2003. 
politiques puis 2) en les mettant en lien avec les débats publics locaux et 3) en faisant le lien entre ces actions politiques et les débats publics et les pratiques spatiales observables à l'échelle de lieux agricoles périurbains. En effet, les interactions entre ces trois composantes des constructions territoriales seront étudiées à partir de la thématique agricole (politique agricole, débats publics à propos de l'avenir de l'agriculture et des espaces agricoles locaux, pratiques spatiales dans les espaces agricoles).

\section{Définitions, méthodes et terrain d'étude}

Cet article propose d'illustrer la complexité du territoire en croisant les notions d'action politique, de débat public et de pratique spatiale qu'il convient donc de définir. Ce croisement a été illustré sur le territoire du Lunellois grâce à une méthodologie originale consistant principalement à identifier des débats publics à partir de l'étude des projets politiques et des journaux locaux qui en témoignent.

\subsection{Quelques définitions}

\section{L'action politique}

L'action peut être définie comme "une manifestation concrète de l'activité de quelqu'un ou d'un groupe» (définition Larousse). Dans ce cadre, les actions politiques analysées ici désignent les manifestations concrètes de la Communauté de communes du Pays de Lunel (groupe étudié) pour faire passer ses idées et imposer ses décisions et, ainsi, gouverner son territoire. En ce sens, ces actions s'apparentent à des actions publiques. C'est la portée politique de ces actions et leurs conséquences sur le contenu des débats publics qui retiendront notre attention, moins le contenu de l'action en tant que tel ${ }^{10}$. Nous nous situons alors dans une vision "politique» du territoire puisque nous nous appuyons au départ sur sa délimitation administrative et politique et sur l'étude des actions qui permettent de qualifier la

10 Pierre Lascoumes et Patrick Le Galès, Gouverner par les instruments, Paris, Les Presses de Sciences Po, 2005. 
politique d'un territoire ${ }^{11}$. Nous souhaitons ensuite examiner ces actions politiques au regard des débats publics qui influencent l'action et qui sont engendrées par elle et des pratiques spatiales qui motivent voire qui réorientent ces actions politiques.

\section{Le débat public}

Le débat public peut être défini au travers des consultations publiques et de son organisation par le politique. Il désigne alors l'émergence de nouvelles formes démocratiques dites «participatives» ou "directes»" ${ }^{12}$. Les débats publics organisés par le politique relaient entre autres des questions d'aménagement et des conflits qui lui sont liés ${ }^{13}$ mais aussi des questions environnementales ${ }^{14}$. Le débat public prend également forme à travers la sphère médiatique $^{15}$, notamment la presse régionale qui a déjà permis d'étudier les conflits dans le périurbain ${ }^{16}$, les questions identitaires en Bretagne ${ }^{17}$ ou encore l'évolution du traitement des risques naturels comme les inondations ${ }^{18}$. Parfois dénoncés

11 Michel Lussault, op. cit.

12 Yves Sintomer et Marie-Hélène Bacqué, La démocratie participative. Histoire et généalogie, Paris, La Découverte, 2011; Laurence Monnoyer-Smith, "Le débat public en ligne : une ouverture des espaces et des acteurs de la délibération?», dans Martine Revel et al., Le débat public : une expérience française de démocratie participative, Recherches, 2007, p. 155-166.

13 Philippe Subra, «À quoi et à qui sert le débat public?», Hérodote, nº 110, 2003, p. 149-155.

14 Daniel Boy, "La place de la question environnementale dans le débat public», Regards croisés sur l'économie, n 6, 2009, p. 48-55; Jean-Luc Pissaloux, «La démocratie participative dans le domaine environnemental », Revue française d'administration publique, $\mathrm{n}^{\text {os }} 137-138,2011$, p. 123-136.

15 Peter Dahlgren, Marc Abélès, Daniel Dayan et Éric Maigret, «L'espace public et les médias : une nouvelle ère?», Hermès, nos 13-14, 1994, p. 243-262.

16 André Torre et Christine Lefranc, "Les conflits dans les zones rurales et périurbaines. Premières analyses de la presse quotidienne régionale», Espaces et sociétés, $\mathrm{n}^{\text {os }} 124-125,2006$, p. 93-110.

17 Thibault Courcelle, «Le rôle de la presse quotidienne régionale bretonne dans la création d'une "identité bretonne" : étude comparative de Ouest-France et du Télégramme», Hérodote, no 110, 2003, p. 129-148.

18 Yves-François Lay et Anne Rivière-Honegger, «Expliquer l'inondation : la presse quotidienne régionale dans les Alpes et leur piedmont (1882-2005)", Géocarrefour, vol. 84, n 4, 2009, p. 259-270. 
comme instrumentalisant ${ }^{19}$, les débats publics locaux n'en donnent pas moins une somme d'informations utiles pour comprendre ce qui se fait et se discute au niveau local. Il s'agit alors plus particulièrement de l'étude des enquêtes publiques lors de la mise en place des schémas de cohérence territoriale (SCoT) et des PLU (plan local d'urbanisme), des comptes rendus des conseils intercommunautaires et communaux qui sont mis à la disposition du public (consultés sur Internet, en mairie ou à l'EPCI) et de la presse quotidienne régionale et communale. Le territoire devient un sujet parmi d'autres au sein de ces débats et notamment lorsque les prises de décision concernent l'aménagement du territoire ou la construction territoriale. Il devient alors support de débat public, débat plus ou moins relayé et alimenté par la sphère médiatique ${ }^{20}$ et plus ou moins conflictuel selon les projets d'aménagement que porte la politique locale ${ }^{21}$. Dans ce cas-là, les actions politiques sont les témoins d'un projet politique plus large. Les débats publics qui accompagnent ce projet politique permettent de le contester ou de le promouvoir. Dans les deux cas, une part de l'argumentation de ces débats s'appuie sur l'adéquation ou l'inadéquation constatée de ce projet politique avec des pratiques spatiales observables dans le territoire.

\section{La pratique spatiale}

La pratique spatiale peut être définie comme "un ensemble de comportements d'un opérateur en relation avec un espace qui constitue pour lui un contexte ${ }^{22} \gg$. Chaque habitant pratique donc un espace qui lui est proche en l'investissant plus ou moins. Guérin-Pace a ainsi montré, à partir de l'étude des déplacements pédestres des citadins, différents types d'investissements, d'une vision simplement utilitaire de l'espace («territoire d'utilisation

\footnotetext{
19 Arnaud Brennetot, "Des bases de données et du débat public dans les régions ", L'espace géographique, tome 40, 2011, p. 133-37.

20 Julien Angelini, «Le débat public comme agir territorial : méthodes qualitatives », Communication et organisation, $\mathrm{n}^{\circ} 38,2010$, p. 171-80.

21 Philippe Subra, "Ce que le débat public nous dit du territoire et de son aménagement", Géocarrefour, no 81, 2006, p. 287-298.

22 Jacques Lévy et Michel Lussault, op. cit., p. 741.
} 
mixte») à son investissement social («territoire investi») ${ }^{23}$. L'espace agricole peut également devenir un «espace de proximité» pour les périurbains. Mais ce qui le différencie d'autres espaces urbains, comme ceux étudiés par Guérin-Pace, est qu'il est également le support d'une activité économique privée qu'une fréquentation accrue peut remettre en cause. Pourtant, en zone périurbaine, les espaces agricoles deviennent le support de multiples pratiques spatiales ${ }^{24}$, de celles de l'agriculteur ${ }^{25}$ à celles du pratiquant de loisirs de plein air, du chasseur ou du cueilleur ${ }^{26}$ en passant par celles de l'aménageur ${ }^{27}$.

La multiplication des pratiques dans l'espace agricole périurbain reflète des comportements individuels (pratiques de loisirs de plein air notamment) mais également collectifs comme l'attachement collectif à un paysage et au cadre de vie qu'il représente et qui est recherché dans le périurbain ${ }^{28}$. Ces pratiques spatiales nouvelles (ou tout du moins renouvelées par des pratiques récentes ${ }^{29}$ ) sont parfois mises en débat localement. Elles peuvent être à l'origine de conflits ${ }^{30}$ : un projet immobilier dégrade le cadre paysager d'un quartier en faisant disparaître des espaces agricoles, un agriculteur interdit le passage sur un chemin fréquenté... Elles peuvent également devenir la raison d'un aménagement visant à canaliser et à protéger ces pratiques : un parcours de santé est construit à l'endroit où des joggeurs couraient déjà,

23 France Guérin-Pace, "Vers une typologie des territoires urbains de proximité», L'espace géographique, no 32, 2003, p. 333-344.

24 Camille Clément, La publicisation des espaces agricoles périurbains : du processus global à la fabrique du lieu, thèse de géographie, Université Montpellier 3, 2012.

25 Christophe Toussaint Soulard, Les agriculteurs et la pollution des eaux. Proposition d'une géographie des pratiques, thèse de géographie, Université Paris 1 La Sorbonne, 1999.

Yvon Le Caro, Les loisirs en espace agricole. L'expérience d'un espace partagé, Rennes, Presses universitaires de Rennes, 2007.

27 Philippe Subra, "Ce que le débat public nous dit du territoire et de son aménagement", op. cit.

28 Rodolphe Dodier, Laurent Cailly, Arnaud Gasnier et François Madoré, Habiter les espaces périurbains, Rennes, Presses universitaires de Rennes, 2012.

29 Olivier Lazzarotti, Les loisirs à la conquête des espaces périurbains, Paris, L'Harmattan, 2000.

30 André Torre et Christine Lefranc, op. cit. 
une route de fort passage de tracteurs est renforcée ou agrandie... Les pratiques spatiales alimentent alors les débats publics locaux et les actions politiques.

\subsection{Matériels et méthodes}

L'objectif visé par cet article est donc de comprendre, grâce à une étude de cas dans les espaces en périphérie de l'agglomération de Montpellier, en quoi les constructions territoriales sont le résultat d'interactions entre actions politiques, débats publics locaux et pratiques spatiales étudiées au travers de la thématique agricole : quelle gestion politique des espaces agricoles et de l'agriculture dans le territoire du Lunellois? Quelles pratiques spatiales s'observent dans ce même territoire? Quelles interactions entre cette gestion politique et ces pratiques spatiales?

Pour répondre à ces questions, le territoire du Lunellois en Languedoc a été étudié à deux échelles.

Premièrement, les actions politiques et les débats publics qui leur sont liés ont été étudiés entre 2002 et 2010 dans la Communauté de communes du Pays de Lunel. Les actions publiques en faveur de l'agriculture ont été recensées grâce à l'étude des comptes rendus de conseils communautaires réalisés durant cette période, complétés par quelques comptes rendus de conseils municipaux (sur des communes concernées par des projets intercommunaux). Afin de saisir comment les décisions prises lors de ces conseils communautaires étaient relayées auprès de la population, une étude des médias locaux (Midi Libre et journal de la Communauté de communes du Pays de Lunel) a été réalisée (toujours de 2002 à 2010) et nous avons également été présente à certaines réunions d'information (une dizaine en tout dans cette période). Enfin, cette étude documentaire a été complétée et enrichie par des entretiens auprès des principaux acteurs ayant pris part à ces événements, principalement des élus et techniciens de la Communauté de communes mais également auprès des responsables d'associations qui animent l'agriculture du Lunellois (association de producteurs, association sur l'histoire 
de la viticulture). En tout, une quinzaine d'entretiens ont été réalisés dans ce cadre.

Deuxièmement, le Lunellois a été étudié au travers de ses pratiques spatiales. Pour cela, nous avons identifié six «lieux agricoles périurbains du Lunellois». Un «lieu agricole périurbain» a été défini comme une zone micro-locale, de surface limitée, composée majoritairement de surfaces agricoles (mais pouvant comporter d'autres types d'occupation du sol ${ }^{31}$ ) qui sont le support de pratiques et de représentations sociales et/ou politiques $^{32}$. Un lieu possède un nom, ce qui le rend identifiable par le collectif et transmissible ${ }^{33}$. Un lieu agricole périurbain possède donc une réalité géographique (situation) et une réalité sociale et politique ${ }^{34}$. Les six lieux agricoles périurbains du Lunellois, de 100 à 200 hectares chacun, ont été repérés grâce à l'étude des actions politiques et des débats publics identifiés dans la première phase de l'étude. Ils ont également été repérés grâce à une démarche "d'observation participante». Celle-ci désigne une démarche d'insertion dans le territoire : vie quotidienne, participation à la vie associative, etc. Une fois repérés, ces lieux ont été étudiés grâce à des entretiens auprès de leur principaux acteurs (agriculteurs, habitants, pratiquants de loisirs de plein air, élus locaux en charge de l'agriculture ou du tourisme). En tout, une vingtaine d'entretiens ont été réalisés dans ce cadre, entretiens complétés par des discussions in situ notamment auprès des pratiquants de loisirs de plein air et par une lecture du paysage de chaque lieu, également étudié à partir de sa cartographie : étude du cadastre pour identifier la propriété, cartographie de sa fréquentation et de son occupation des sols.

Cette étude du Lunellois à deux échelles a d'abord permis de décrire les principales caractéristiques du Lunellois et les actions politiques mises en œuvre entre 2002 et 2010 sur la thématique agricole. Cinq débats publics en interaction directe avec ces actions politiques ont alors été identifiés. Ensuite, l'étude des six

31 Le Caro, Yvon, op.cit.

32 Jacques Lévy et Michel Lussault, op. cit., p. 555.

33 Hannah Arendt, La crise de la culture, Paris, Gallimard, 1989.

34 Camille Clément, op.cit. 
lieux agricoles périurbains a permis d'identifier les connexions entre ces débats publics, les actions politiques et les pratiques spatiales observables sur le territoire. Ainsi, si une grande partie des pratiques spatiales observables dans l'espace agricole du Lunellois se retrouvent dans les actions politiques et les débats publics qui les animent, d'autres ne s'y retrouvent pas. Enfin, c'est à partir de ce constat que nous nous sommes interrogée sur la manière dont les pratiques spatiales pourraient être prises en compte dans l'étude de la complexité des constructions territoriales.

\section{Résultats}

2.1. Le Lunellois : un territoire périurbain en crise d'identité

Le Lunellois est un territoire situé entre Montpellier et Nîmes et organisé autour de la ville de Lunel (24044 habitants). La Communauté de communes du Pays de Lunel (CCPL) était composée au moment de l'étude de 13 communes réparties sur une bande Nord-Sud entre les deux agglomérations du Nîmes et de Montpellier pour un total de près de 46000 habitants $^{35}$ (voir la figure).

$\overline{35}$ Institut National de la Statistique et des Études Économiques (INSEE), Statistiques sur l'EPCI de la CC du Pays de Lunel, 2011, [en ligne] http://www. insee.fr/fr/themes/dossier_complet.asp?codgeo=EPCI-243400520, consulté en septembre 2014. 
Figure :

Localisation du secteur d'étude

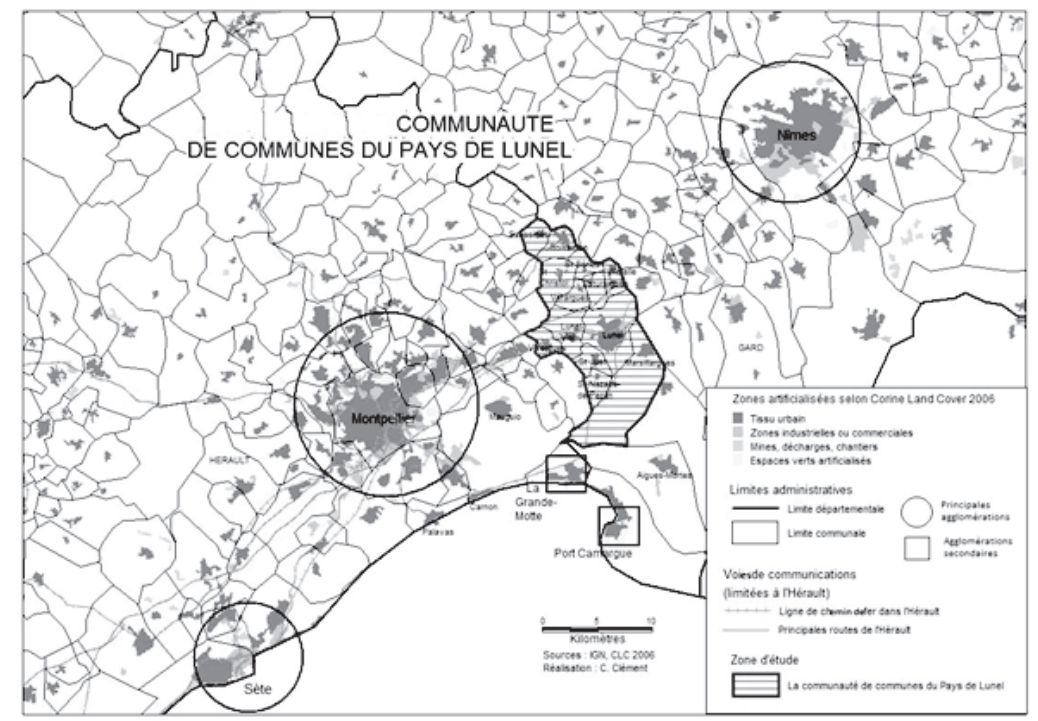

Les espaces agricoles couvrent environ $53 \%$ du territoire total (données CCPL). $56 \%$ des 344 exploitations du territoire recensées en 2010 sont individuelles mais ne couvrent que $34,2 \%$ de la surface agricole utile (SAU) totale (soit 2083 hectares). La CCPL a connu une concentration des exploitations avec un total de 344 exploitations en 2010 pour 488 en 2000 et 738 en $1988^{36}$. Selon les données du Recensement général agricole (RGA) de 2010, $22 \%$ des 344 exploitations actuelles de la CCPL sont concernées par les céréales qui couvrent un quart de la SAU. La vigne concerne quant à elle 57,3\% des exploitations et $27,1 \%$ de la SAU totale.

Le Nord de la CCPL est la zone de production du muscat de Lunel, reconnu depuis le Moyen-Âge où il était servi à la cour

36 Toutes les données du dernier Recensement Général Agricole par commune, canton, département et région, sont accessibles sur tableur du site du ministère de l'agriculture et de l'agreste : [en ligne] http://www.agreste.agriculture.gouv.fr/ recensement-agricole-2010/. 
pontificale d'Avignon ${ }^{37}$. Le reste du territoire viticole est occupé par des vignes sans appellation d'origine contrôlée (AOC) et des vignes sous AOC Côteaux du Languedoc Saint-Christol. La partie centrale de la Communauté de communes est marquée par une forte urbanisation et la présence de nombreuses voies de communication. Ainsi, du Nord au Sud, se succède sur la CCPL l'autoroute A9 qui relie Barcelone à Lyon, la voie de chemin de fer entre Perpignan et Nîmes, la Route Nationale 113 et le canal Philippe Lamour construit dans les années 1950 afin de rendre irrigable toute la plaine Nord de Mauguio. Cette zone de circulation intense accueille les principaux espaces économiques de la CCPL avec 11 des 14 zones d'activité du territoire situées dans cette partie du territoire ${ }^{38}$. Celle-ci est par conséquent la moins agricole et la plus artificialisée. La partie Sud du territoire voit la vigne quasiment disparaître au profit de grandes cultures et de vergers. Dans cette zone, l'agriculture est dominante et organisée autour de grands domaines, les mas, qui cultivent principalement de grandes cultures maraîchères, des vergers et des manades (élevage de taureaux camarguais). Le Sud du territoire est aussi marqué par la présence d'espaces naturels remarquables du point de vue écologique. Ainsi la partie Sud-Ouest de la CCPL est sous dominance maritime car elle borde l'Étang de Mauguio (ou Étang de l'Or). Elle accueille des classements ZNIEFF (zone naturelle d'intérêt écologique, floristique et faunistique), ZICO (zone importante pour la conservation des oiseaux) et Natura 2000. Cette zone Sud est quasiment entièrement inondable selon les documents de prévention du risque inondation de la Communauté de communes.

La CCPL est en effet soumise à un fort risque inondation du fait de son principal fleuve, le Vidourle, qui fonctionne sous régime méditerranéen avec des crues violentes et soudaines appelées localement «les vidourlades». Ainsi, en 2002, mais aussi en 2003 et 2005, de tels épisodes ont eu lieu touchant principa-

\footnotetext{
37 Claude Raynaud, "Histoire d'un nectar : le muscat de Lunel», Études héraultaises, hors-série, 2011, p. 31-47.

38 Schéma de Cohérence Territoriale (SCoT) du Pays de Lunel, Communauté de communes du Pays de Lunel, 2006, p. 76.
} 
lement Lunel et le sud du territoire (en 2002 sur les départements de l'Hérault, du Gard et du Vaucluse il y a eu 24 morts).

La Communauté de communes du Pays de Lunel se situe donc à la rencontre de deux grandes aires d'héritages culturels : les traditions camarguaises d'un côté et l'histoire viticole de l'autre. Ce croisement constitue l'un des fondements identitaires de ce territoire administratif ${ }^{39}$. À cela, nous pouvons ajouter un troisième fait marquant, les frontières du Lunellois actuel : la limite administrative entre le Gard et l'Hérault marquée par le fleuve Vidourle.

La situation géographique et l'histoire récente du Lunellois et de sa Communauté de communes soumettent ce territoire à des problèmes d'identité celui-ci se situant :

- entre l'urbain et le rural, car il connaît un processus de périurbanisation;

- entre le Gard et l'Hérault;

- entre coteaux et littoral, car le Nord du territoire est plus vallonné et est principalement occupé par de la vigne tandis que le Sud est en bordure d'étang et se caractérise par de grands domaines spécialisés dans les grandes cultures ou les vergers (parfois des prairies avec la présence d'élevage de taureaux);

- entre héritage viticole (même si aujourd'hui l'activité viticole se concentre dans le Nord du territoire) et traditions camarguaises venant du Sud et de l'Est.

Dans cette recherche d'identité, l'agriculture joue un rôle majeur. Le Lunellois possède une histoire agricole riche qui est aujourd'hui mobilisée dans des projets patrimoniaux (pôle œnotouristique, exposition nommée "vin, vigne, vigneron" qui retrace l'histoire viticole du territoire). Comment les politiques intercommunautaires mises en ouvre entre 2002 et 2010 ontelles traité des questions agricoles? Comment cela a-t-il été relayé dans le débat public via la communication de ces actions politiques dans les médias locaux?

39 Ibid. 
2.2. Actions politiques et débats publics : le cas de l'agriculture dans la Communauté de communes du Pays de Lunel

Trois projets politiques principaux concernant l'agriculture ont été identifiés suite au travail de terrain dans le Lunellois de 2002 à 2010. Il s'agit de la rédaction du SCoT entre 2002 et 2006 [composé d'un diagnostic de territoire, d'un projet d'aménagement et de développement durable (PADD) et d'un document d'orientation générale (DOG), les trois étapes de construction du schéma], de la politique de développement des circuits courts [installation de nouveaux maraîchers, mise en place d'un marché de producteurs, convention avec le Centre d'initiatives pour valoriser l'agriculture et le milieu rural (CIVAM) et la Société d'aménagement foncier et établissement rural (SAFER)] et de la mise en œuvre d'un pôle œnotouristique ouvert au public depuis octobre 2013 mais qui a débuté début 2005. Le tableau décrit les principaux événements qui ont animé ces trois projets. Ceux-ci ont été mis en regard avec les principaux événements identifiés dans la presse régionale et ayant impacté (de près ou de loin) cette politique agricole. Ainsi, cinq débats publics en lien avec la politique agricole de l'intercommunalité lunelloise ont été mis à jour sur la période 2002-2010. 


\section{Tableau :}

\section{Actions politiques et débats publics dans le Lunellois entre 2002 et 2010}

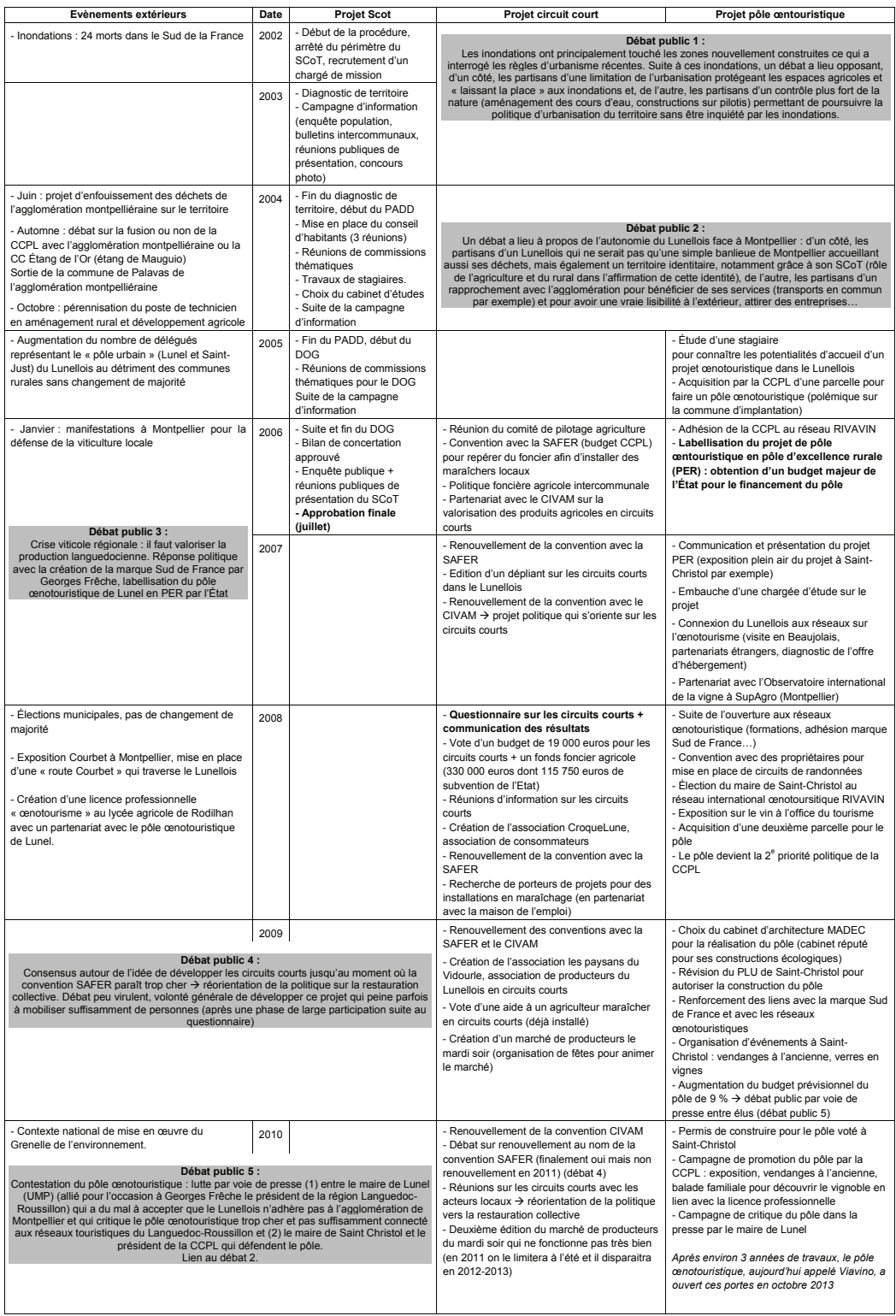


Cette reconstitution des principaux événements et actions politiques qui se sont déroulés entre 2002 et 2010 dans la Communauté de communes du Pays de Lunel montre les liens existant entre actions politiques et débats publics à l'échelle locale. Premièrement, le SCoT, voté en 2006, limite les possibilités d'artificialisation dans le Lunellois. Les objectifs visent à une réduction d'au moins $35 \%$ et d'au plus $50 \%$ de la consommation d'espace pour l'urbanisation selon les communes. Dans les faits, cela limite l'artificialisation des sols à l'échelle intercommunale entre 115 et 125 hectares pour l'habitat, entre 90 et 125 hectares pour les zones économiques et entre 10 et 20 hectares pour les équipements publics, et ce, pour 10 ans. Les PLU et plan d'occupation des sols (POS) de chaque commune doivent être conformes à ces fourchettes de surfaces constructibles ${ }^{40}$. Ces restrictions limitent l'étalement urbain (notamment dans les zones inondables) et protègent les espaces agricoles. Le tableau montre que deux débats publics ont directement influencé cette politique. Les inondations de 2002 ont marqué voire traumatisé la population (débat 1 dans le tableau). Les médias ont relayé la solidarité qui s'est créée entre les habitants suite à ces événements. Au même moment, le diagnostic du SCoT était en cours et le traumatisme qui a suivi ces inondations a facilité la mise en place de cette politique alors même que le territoire se périurbanise, ce qui entraîne de fortes demandes en terme d'habitat, notamment pavillonnaire $^{41}$. Les débats autour des aménagements à réaliser suite à ces inondations ont été vifs. Des experts ont préconisé l'agrandissement du lit du Vidourle (déplacement des digues) avec des zones plus basses dans les digues, sorte de déversoirs qui inonderaient prioritairement les zones agricoles pour protéger les villages. Certains ont salué cette politique, d'autres, notamment les habitants des zones concernées par ces déversoirs, l'ont critiquée (syndrome NIMBY ${ }^{42}$ ) jugeant qu'il fallait trouver une solution pour qu'il n'y ait plus jamais ce type d'inondation.

\footnotetext{
40 Ibid.

41 Rodolphe Dodier et al., op. cit.

42 Michael Dear, "Understanding and Overcoming the NIMBY Syndrome", Journal of the American Planning Association, vol. 58, n 3, 1992, p. 288-300.
} 
Cette politique de protection foncière s'est également affirmée dans un contexte de lutte contre l'intégration du Lunellois à l'agglomération montpelliéraine (débat 2 dans le tableau). Celle-ci a été particulièrement vive en 2004 et a également orienté la politique du SCoT. À cette époque, l'agglomération montpelliéraine tente des rapprochements avec les Communautés de communes de l'Étang de l'Or et du Pays de Lunel. Au même moment, le maire de Palavas entre en conflit ouvert avec Montpellier, ce qui l'amène à quitter l'agglomération pour rejoindre celle de l'Étang de l'Or ${ }^{43}$. Des tractations, relayées par les journaux locaux, ont lieu pour savoir si les Communautés de communes de l'Étang de l'Or et du Pays de Lunel vont fusionner pour mieux peser face à l'agglomération montpelliéraine. Le Lunellois choisit de rester indépendant notamment pour des questions politiques (l'Agglomération de Montpellier est socialiste, les principales villes de la côte sont à droite ainsi que la ville de Lunel, en revanche la Communauté de communes du Pays de Lunel est également socialiste) ${ }^{44}$. Pour affirmer son caractère indépendant, le Lunellois profite du SCoT et affirme l'identité rurale et agricole de son territoire : "un espace de respiration" entre les agglomérations de Nîmes et Montpellier ${ }^{45}$. Cette recherche d'identité rurale passe par la création de vice-présidents de la CCPL en charge de «l'identité rurale» mais aussi par une politique de protection du foncier agricole promue au niveau national. Elle passe également par le financement de projets qui promeuvent cette identité rurale et agricole. Le conventionnement avec la SAFER et le CIVAM afin de développer les circuits courts dans le Lunellois (aide à l'installation de maraîchers,

43 Cette décision a eu des conséquences importantes sur la politique de l'agglomération de Montpellier. Ainsi, aujourd'hui, le tramway de l'agglomération ne va pas jusqu'à la mer mais s'arrête à Pérols, commune, non littorale, limitrophe de Palavas et faisant partie de l'agglomération.

44 L'Agglomération de Montpellier est socialiste mais "tendance Frêche», l'exprésident de la région Languedoc-Roussillon, aujourd'hui décédé, mais très décrié de son vivant au sein du parti socialiste. La Communauté de communes du Pays de Lunel est dirigée par une majorité socialiste plus classique qui s'oppose dans les faits aux élus proches de Georges Frêche.

Schéma de Cohérence Territoriale (SCoT) du Pays de Lunel, op. cit. 
communication sur les producteurs en vente directe et incitation auprès des producteurs à faire de la vente directe) ainsi que la création d'un pôle œnotouristique sont les deux projets en lien avec l'agriculture lunelloise et, totalement ou en partie, financés par la CCPL.

Ces deux projets sont donc directement issus des réflexions initiées dans le cadre du SCoT ce que montre clairement la chronologie mise en évidence dans le tableau. De même que pour le SCoT, ces deux projets sont directement des actions politiques en lien avec les débats publics de l'époque. En 2006, une crise viticole secoue le Languedoc et de nombreuses manifestations ont lieu à Montpellier pour défendre la viticulture régionale et pour demander des aides à l'État. Pour répondre à cette crise, la Région Languedoc-Roussillon mise sur la valorisation des produits locaux. C'est dans ce contexte qu'est créée la marque "Sud de France». Lancée par Georges Frêche, alors président de la Région Languedoc-Roussillon, cette marque vise à valoriser les produits de la région, notamment à l'étranger. La création de cette marque est l'aboutissement d'un travail de revalorisation de la production viticole du Languedoc déjà initiée depuis quelques années (réencepagement, productions de vins de cépages, AOC, etc.). Déjà en 2005, le Lunellois avait perçu l'importance de ce changement d'image et avait financé un stage pour évaluer le potentiel œnotouristique du territoire. Le maire de Saint-Christol est particulièrement actif dans cette politique. Maire de la commune portant le nom d'une appellation (le Saint-Christol est l'une des sous-appellations de l'AOC Côteaux du Languedoc), il croit énormément dans le potentiel œenotouristique du territoire connu pour son muscat. En 2006, avec d'autres élus de la CCPL, il décide de profiter de l'appel d'offres de l'État sur les pôles d'excellence rurale pour proposer un projet de pôle œnotouristique. Cet appel d'offres est incitatif : il permet d'obtenir des financements importants de l'État pour construire un projet pour le territoire tout en affirmant le caractère rural du Lunellois. Le projet est financé en 2006 (labellisation) et son budget est complété par des fonds venant de la Communauté de 
communes, du Conseil général et, dans une moindre mesure, de la Région. En effet, cette dernière voit d'un mauvais œil ce projet, source d'affirmation pour un territoire qui, selon les déclarations de Georges Frêche à cette époque (il est aujourd'hui décédé), devrait être intégré à l'agglomération montpelliéraine. Ainsi, le pôle œnotouristique devient également une source de polémique animant le débat public 2 déjà évoqué. Les points de vue favorables ou hostiles au projet œnotouristique s'expriment dans le Midi Libre. Le maire de Lunel, partisan de la fusion avec Montpellier ou avec la Communauté de communes de l'Étang de l'Or, est également frustré d'avoir vu le projet se concrétiser en dehors de sa commune. Il juge le projet trop cher pour une si petite Communauté de communes. Par ailleurs, la CCPL et le maire de Saint-Christol financent des opérations de communication sur la pertinence du projet onotouristique du Lunellois (exposition sur l'histoire du projet et de la viticulture dans le Lunellois, articles de journaux, réunions publiques d'information sur le projet).

Le projet de valorisation des circuits courts est beaucoup moins polémique dans le Lunellois. Un questionnaire datant de 2008 a mis en évidence une demande forte mais non comblée pour une alimentation de proximité. La Communauté de communes décide de financer deux opérations par conventionnement, l'un avec la SAFER afin de repérer des parcelles pour installer de nouveaux maraîchers pour répondre à cette demande et ainsi mettre en œuvre une politique foncière agricole intercommunale, l'autre avec le CIVAM de l'Hérault (CIVAM34) afin de réaliser des études sur l'offre existante en circuits courts et développer des outils de communication et de développement de ce mode de commercialisation. Les initiatives dans ce sens se multiplient. En 2008 une association de consommateurs est créée et des porteurs de projets de maraîchage sont sélectionnés par le CIVAM34 et la maison de l'emploi. En 2009, une association de producteurs est créée et un marché de producteurs voit le jour. Mais le conventionnement avec la SAFER coûte cher et la politique foncière est difficile à mettre en ouvre. Les porteurs de 
projets sérieux sont peu nombreux et, selon le technicien en charge de l'agriculture à la CCPL, les domaines que la SAFER propose au rachat pour la CCPL sont trop grands et trop chers pour la Communauté de communes. Cela créera une polémique relayée par les médias locaux (débat public 4 dans le tableau) et, finalement, le conseil communautaire décidera en 2011 de ne pas renouveler son conventionnement avec la SAFER. Du même coup, la CCPL abandonnera sa politique foncière agricole intercommunale trop coûteuse dans une période de crise économique qui limite de plus en plus les aides de l'État et le financement sur ressources propres. En contrepartie, elle privilégiera la politique moins coûteuse de promotion des circuits courts (le conventionnement avec le CIVAM est maintenu) qui se réoriente sur la restauration collective au moment où le Grenelle de l'environnement oblige les collectivités territoriales à introduire des produits bio ou locaux à hauteur de $20 \%$ dans la restauration collective avant 2012 .

L'étude des actions politiques et des débats publics concernant l'agriculture dans le Lunellois entre 2002 et 2010 a montré leurs fortes interactions. Ainsi certaines polémiques extérieures comme la crise viticole, les inondations survenues en 2002 ou les débats autour du Grenelle de l'environnement ont directement influencé les choix et actions politiques initiés dans le Lunellois (pôle œnotouristique, limitation de l'étalement urbain, réorientation du projet de circuits courts sur la restauration collective). Ces mêmes actions ont elles-mêmes généré des débats publics. Le premier concerne la place du Lunellois dans l'agglomération montpelliéraine : faut-il s'en rapprocher ou garder son indépendance et une identité forte? Sur cette question, le caractère rural et agricole du territoire joue un fort rôle identitaire. Il n'est pas le seul : le territoire joue aussi sa différence sur le taux d'imposition jugé beaucoup trop élevé à Montpellier. Les polémiques se focalisent alors sur la pertinence d'un projet de pôle onotouristique concernant uniquement le Lunellois et le choix d'une politique foncière agricole intercommunale qui se révèle de plus en plus complexe à mettre en place à cette échelle. Ainsi des visions 
différentes du territoire s'affrontent et sont relayées par des actions politiques qui sont largement influencées par les taux d'imposition locaux qu'elles entraîneraient. Ces actions politiques et ces débats publics sont également à mettre en lien avec les pratiques des habitants du Lunellois et notamment leur pratiques spatiales observables dans des lieux agricoles périurbains.

2.3. Actions politiques et pratiques spatiales : une rencontre à l'échelle du lieu agricole périurbain

La sélection et l'identification de lieux agricoles périurbains constituent l'un des pans majeurs de l'étude des actions politiques et des débats publics dans le Lunellois. Certains lieux précis du Lunellois ont directement fait l'objet de ces débats. Ainsi, le débat sur les conséquences des inondations de 2002 a été particulièrement fort à Marsillargues où un projet de déversoir a été proposé. Marsillargues a été le village le plus inondé en 2002 et ce projet a été très mal accueilli par les habitants. Une grande partie d'entre eux ne voulaient plus que de telles inondations puissent exister. Ils défendaient alors une position $\mathrm{du}$ "plus jamais ça» non seulement dans le village mais également dans les terres agricoles qui sont restées plusieurs semaines sous l'eau avant que des opérations de pompages soient menées. Les zones d'enjeux du SCoT sont elles aussi mises en exergue par l'action publique : elles bénéficient de règles particulières quant à leur protection de l'urbanisation. Enfin, le lieu de construction du pôle œnotouristique a, lui également, fait l'objet de nombreux débats. Prévu successivement à Lunel puis à Marsillargues, c'est finalement à Saint-Christol qu'il a été construit grâce au dynamisme de son maire et de sa cave coopérative et grâce à la présence de l'appellation Saint-Christol ${ }^{46}$.

46 Marsillargues était la commune la plus viticole au début du siècle dernier au moment où elle accueillait la plus grande cave coopérative d'Europe en termes de production. Aujourd'hui seulement $6 \%$ de la surface communale est viticole et la cave coopérative a été détruite. C'est pour protéger ce patrimoine viticole que certains acteurs soutenaient le projet d'y implanter le pôle œnotouristique. 
Les autres débats, sans vraiment s'appuyer sur une zone particulière du Lunellois, témoignent de certaines pratiques spatiales en augmentation sur le territoire. Ainsi, les journaux locaux ont de plus en plus parlé de la multiplication de l'offre de loisirs de plein air dans ce territoire en périurbanisation : implantation d'une station de canoë et d'une via ferrata à la Roque-de-SaintSériès, création d'une zone d'accrobranche et d'une piste d'atterrissage d'avions miniatures à Saint-Christol ou encore création d'un parcours de santé à Saturargues, un autre à Lunel. Enfin, le développement d'une politique de valorisation des circuits courts a mis en valeur les exploitations pratiquant la vente directe (maraîchers, viticulteurs, horticulteurs, etc.).

C'est à partir de ces éléments que six lieux agricoles périurbains ont été définis et étudiés en détail ${ }^{47}$ au travers de différentes caractéristiques (propriété, occupation du sol, proximité à l'urbanisation...) et par les pratiques spatiales qui les animent.

$\mathrm{La}$ «zone du déversoir de Marsillargues» qui accueille également aujourd'hui des jardins familiaux a vu sa fréquentation accrue au moment des travaux et des polémiques qui accompagnaient sa construction. Depuis la fin des travaux, les digues du Vidourle restent fréquentées pour la promenade, les joggings, les piqueniques ou, en période de crue, pour surveiller la montée du Vidourle.

La «zone enjeux n ${ }^{\circ} 1 \mathrm{du}$ SCoT» n’a fait l'objet de débats que dans le cadre du SCoT. Peu de pratiques spatiales, autres que celles des agriculteurs de la zone, sont observables sur place. Cette zone, qui n'a pas vraiment de réalité sociale, ne correspond pas à notre définition d'un lieu agricole périurbain mais désigne une coupure d'urbanisation entre deux villages. C'est également la zone prévue pour un contournement routier de la ville de Lunel. Les parcelles sont en grande proportion privées avec des jardins d'agrément clôturés qui côtoient des zones agricoles (grandes cultures, vergers) plus classiques.

Le lieu de construction du pôle œnotouristique a été ouvert aux espaces alentours pour y intégrer des circuits de randonnées

47 Clément, Camille, op. cit. 
déjà existants lors de l'étude contrairement au pôle en lui-même dont les travaux de construction n'avaient pas encore commencé au début de l'étude (inauguration du pôle en octobre 2013). Il s'agit alors du «lieu des croix de Malte" pour désigner le nom de la randonnée qui parcourt le village de calvaire en calvaire. Ce circuit a été programmé pour passer à proximité de producteurs en vente directe. Les paysages sont valorisés (haies d'oliviers) et, aux dires des agriculteurs sur place, des groupes de randonneurs à pied et à vélo passent régulièrement. Cette zone fait également l'objet de vendanges à l'ancienne où des volontaires viennent cueillir le raisin à la main dans une région où la machine à vendanger est systématiquement utilisée depuis une dizaine d'années.

Les trois autres lieux d'étude ne correspondent pas directement à un débat public. "La tour de Farges" désigne un domaine viticole produisant du muscat de Lunel. Situé à proximité des voies de communication majeures (autoroute, future ligne TGV qui va couper le domaine en deux, voie de chemin de fer), ce domaine a néanmoins choisi de développer une politique "patrimoniale» suite, notamment, à la mise en place de la route Courbet qui passe par son mas peint par cet artiste ${ }^{48}$. Il fait de la vente directe, accueille des événements culturels dans le mas et travaille sur l'aspect paysager du domaine : haies d'oliviers, rosiers en bas des rangées ${ }^{49}$. Ce domaine étant proche de Lunel et de Lunel-Viel (village voisin), de nombreux habitants s'en servent de lieu de pratiques de loisirs de plein air. Ainsi, le propriétaire a confirmé croiser de nombreux randonneurs, joggeurs, cyclistes, cueilleurs de mûres sur son domaine. Il a également précisé que des jeunes viennent régulièrement faire la fête à un endroit précis de son domaine en laissant leurs déchets sur place (c'est luimême, au nom de sa "politique patrimoniale», qui les ramasse, le service municipal étant trop peu réactif).

\footnotetext{
48 «Vue de la Tour de Farges» par Courbet, 1857.

49 Historiquement, cette pratique permettait de repérer si la vigne allait être attaquée par certains parasites, les rosiers en étant les premières victimes. Á présent le viticulteur affirme que c'est uniquement pour l'esthétique qu'il maintient la présence de ces rosiers.
} 
"La Roque de Saint-Sériès» est une zone naturelle remarquable bordée par une zone de garrigue et une zone agricole dominée par la viticulture et les grandes cultures. C'est une zone de relief dans une région peu escarpée. Ici, des falaises se jettent dans le Vidourle et font l'objet de pratiques d'escalade et de via ferratta. Le Vidourle est également fréquenté à cet endroit par des pratiquants de canoë. Le lieu est donc dominé par des pratiques de loisirs de plein air qui côtoient les pratiques agricoles d'entretien et de récolte des parcelles à proximité.

Enfin, «les cabanes de Marsillargues» composent un lieu totalement différent des précédents. Situé à l'extrême Sud du territoire et sous influence maritime, ce lieu est habité à l'année depuis environ trois décennies par des personnes travaillant majoritairement à Montpellier ou dans le Lunellois. Traditionnellement, ces cabanes servaient à loger les chasseurs et pêcheurs durant les périodes dédiées à ces activités. Aujourd'hui ces zones sont toujours des lieux de chasse et de pêche. Elles sont également protégées pour leur écosystème particulier (Natura 2000) ce qui attire des ornithologues amateurs tandis que les paysages de bords d'étangs attirent les pratiquants de loisirs de plein air, notamment des cyclistes qui profitent des faibles dénivellations. L'espace agricole jouxte cette zone mais en est séparé par une route nationale. Composée de grandes cultures maraîchères et d'une manade (élevage de taureaux camarguais), cette zone est beaucoup moins fréquentée sinon durant les fêtes votives qui amènent la population à se déplacer au plus proche des manades pour y observer les taureaux qui vont par la suite concourir dans les courses camarguaises.

Ce dernier lieu permet ainsi de mettre en lumière une composante identitaire importante du Lunellois mais qui n'est pas ressortie de manière significative dans les autres lieux étudiés : les traditions camarguaises. Appelé également «le grand Pays de la Petite Camargue», le Pays Lunellois pratique la course camarguaise. Tous les villages du Lunellois (y compris Lunel) possèdent des arènes où se pratique ce sport qui ne consiste pas en la mort du taureau comme dans les corridas mais dans un jeu où le razeteur 
(homme habillé en blanc) tente de saisir une cocarde placée entre les cornes du taureau. Ces jeux revêtent une grande importance dans ce territoire, ils animent l'été et les week-ends du printemps à l'automne ${ }^{50}$. Aujourd'hui, ces traditions populaires datant du $\mathrm{XIX}^{\mathrm{e}}$ siècle sont en partie menacées entre autres à cause des règles de sécurité de plus en plus strictes dans l'encadrement de ce type de manifestation. Régulièrement, les médias locaux se font l'écho de la peur des habitants de voir disparaître ces traditions. En témoigne cet extrait d'un article du Midi Libre publié le $1^{\text {er }}$ août 2011 :

La jeunesse porte le deuil de ses traditions : un Bajan mécontent est un Bajan qui s'exprime ${ }^{51}$. C'est ce qui s'est passé samedi matin lors du défilé d'ouverture de la fête. Habillés en noir, portant des cercueils et brandissant une banderole signifiant le deuil de leurs traditions, les jeunes ont parcouru la ville pour protester contre un programme des festivités 2011 particulièrement allégé.

La fréquentation accrue de ces fêtes liée à la périurbanisation du territoire et à une consommation excessive d'alcool rendent dangereuse la présence de taureaux dans les villages (des taureaux peuvent circuler en liberté dans le village mais également aux abords des routes reliant les manades au village). Cette dangerosité est renforcée par la durée jugée parfois excessive de ces fêtes votives qui peuvent s'étendre sur dix jours dans certains villages. Les préfectures obligent donc les communes à prendre des mesures face à ces risques de troubles à l'ordre public : réduction de la durée des fêtes, allègement du programme, ce qui suscite la colère d'un certain nombre d'habitants et un débat public vif.

Ainsi, tandis que l'étude des cinq premiers lieux au travers des pratiques spatiales qui s'y expriment (notamment en termes de loisirs de plein air et de présence des agriculteurs sur place) a permis de confirmer les débats publics identifiés à partir de

50 Rémi Allemand, Sylvain Rouanet et Jacques Gleyse, «La fête du taureau ou des taureaux dans la fête?», Sociétés, $n^{\circ} 107,2010$, p. 73-86; Jean-Pierre Chaze, «La course camarguaise : un sport ou un jeu traditionnel?», Corps et culture, $\mathrm{n}^{\circ} 4,1999$, p. 15.

51 Un Bajan est le surnom donné aux habitants de Marsillargues, l'un des villages du Lunellois. Le terme vient du languedocien bajan qui signifie littéralement fou ou nigaud. 
l'étude des actions politiques à l'œuvre dans le Lunellois, le sixième lieu a mis en évidence un sixième débat public. Ce débat sur les traditions taurines dans le Lunellois concerne l'agriculture du territoire (élevage) mais n'est relayé par aucun projet ou action politique d'envergure de la part de la Communauté de communes. Pourtant des projets de patrimonialisation de ces traditions (pour les protéger) ou de maintien des manades sur le territoire (politique foncière) auraient pu être menées par la CCPL. Ces traditions camarguaises sont également à l'origine d'une forte appropriation culturelle sur laquelle la CCPL aurait pu s'appuyer pour se distinguer des régions urbaines proches. Ce manque d'implication des pouvoirs publics dans la valorisation des traditions camarguaises est à relativiser face à la part importante du budget culturel des communes qui est consacrée à ces événements taurins prenant place dans des espaces précis : les arènes mais également les prés où les habitants se déplacent pendant la fête votive afin de voir les taureaux qui seront dans l'arène l'aprèsmidi d'où l'identification de ces prés comme de nouveaux lieux agricoles périurbains. Néanmoins, nous pouvons nous demander pourquoi ces traditions n'ont pas été mobilisées dans la construction d'une identité lunelloise (par la CCPL) et si elle le sera dans l'avenir.

L'étude des actions politiques et des débats publics associés ne permet donc pas d'identifier l'ensemble des ressorts des constructions territoriales à l'œuvre dans le Lunellois. En revanche, leur mise en lien avec l'étude des pratiques spatiales observables dans des lieux agricoles périurbains a permis d'identifier un autre débat public structurant pour le territoire mais ne faisant l'objet d'aucun projet d'ampleur par la CCPL. C'est donc l'étude du triptyque action politique, débat public et pratique spatiale qui permet de saisir la complexité des constructions territoriales.

\section{Interprétation : la complexité des constructions territoriales au prisme des pratiques spatiales}

Nous avons vu en introduction que les définitions du territoire étaient nombreuses. Dans cette étude de cas, nous avons d'abord 
étudié le Lunellois au travers de ses actions politiques. L'objectivation du territoire a donc d'abord été politique et administrative $e^{52}$, et non économique ${ }^{53}$ ou culturelle ${ }^{54}$. Ces actions portées par la Communauté de communes sur la thématique agricole ont été complétées par les interactions qu'elles entretiennent avec des débats publics locaux. L'objet central de cet article et du travail de thèse qui en est à l'origine ${ }^{55}$ est le lien ente actions politiques et débats publics, d'une part, et pratiques spatiales observables dans les espaces agricoles de ce territoire, d'autre part. Ces pratiques spatiales n'ont pu être étudiées qu’à l'échelle du lieu agricole périurbain qui est devenu la plus petite unité spatiale permettant de saisir la complexité du territoire. En effet, il s'est avéré que l'échelle administrative des treize communes étudiées est trop large pour saisir la complexité locale des pratiques spatiales pourtant à l'origine du territoire vécu ${ }^{56}$.

Cette étude de cas permet alors d'illustrer deux niveaux de complexité du territoire en s'appuyant sur la définition du terme proposée par Edgar Morin :

Au premier abord, la complexité est un tissu (complexus : ce qui est tissé ensemble) de constituants hétérogènes inséparablement associés : elle pose le paradoxe de l'un et du multiple. $\mathrm{Au}$ second abord, la complexité est effectivement le tissu d'événements, actions, interactions, rétroactions, déterminations, aléas, qui constituent notre monde phénoménal ${ }^{57}$.

Dans un premier temps, actions politiques et débats publics peuvent être considérés comme deux «constituants hétérogènes inséparablement associés» du territoire. Les actions politiques doivent être relayées par des débats publics de qualité, ce qui nécessite la présence de médias locaux reconnus et de l'organisation

52 Michel Lussault, op. cit.

53 Hervé Gumuchian et Bernard Pecqueur, op. cit.

54 Bernard Debarbieux, op. cit.

55 Camille Clément, op. cit.

56 Armand Frémont, La région, espace vécu, Paris, Presses universitaires de France, 1976; Alexandre Moine, «Le territoire comme un système complexe : un concept opératoire pour l'aménagement et la géographie», L'espace géographique, $\mathrm{n}^{\circ} 35,2006$, p. 115-132.

57 Edgar Morin, Introduction à la pensée complexe, Paris, ESF, 1990, p. 17. 
par les instances politiques de consultations publiques voire de co-construction d'actions politiques avec les habitants. Cela suppose également, mais c'est un autre débat, une bonne santé du système démocratique en place. De la même manière, les débats publics locaux ont besoin d'être régulièrement alimentés par des actions politiques. Ainsi, dans la Communauté de communes du Pays de Lunel, actions politiques et débats publics interagissent :

- soit parce que le débat public influence l'action politique : le débat suite aux inondations influence la mise en œuvre du SCoT, les débats autour du Grenelle de l'environnement réorientent la politique locale de circuits courts vers la restauration collective;

- soit parce que l'action politique influence le débat public: l'échelle de la CCPL est finalement considérée comme peu pertinente pour la mise en œuvre d'une politique foncière agricole intercommunale, ce qui renforce le débat sur l'intégration ou non du Lunellois à une autre intercommunalité;

- soit enfin parce que les deux s'inter-influencent réciproquement. C'est le cas du pôle onotouristique imaginé dans le contexte de débat public sur la crise viticole puis mis en débat pour savoir si l'échelle lunelloise est la bonne pour ce type de projet.

Mais l'étude de ces deux composantes du territoire ne prend pas en compte les habitants du territoire que l'on peut pourtant considérer comme les «maillons de base» de la construction territoriale. Pour les prendre en compte, une étude des pratiques spatiales observables dans le territoire est nécessaire. Pour cela, l'étude du lieu agricole périurbain devient nécessaire pour dépasser l'objectivation initiale du territoire qui s'est faite par le politique (et non par le culturel qui aurait axé beaucoup plus rapidement l'étude du territoire sur le territoire vécu de ses habitants et aurait identifié plus rapidement l'importance des traditions camarguaises mais peut-être au détriment d'une autre 
composante du territoire). Ici, l'étude approfondie de six lieux agricoles périurbains a permis de mettre en évidence une composante culturelle importante et fondatrice du territoire que les actions politiques ne montraient par clairement : les traditions camarguaises. Le lieu est alors devenu la seule échelle d'analyse permettant de saisir sur un même espace la pratique spatiale, le débat public et l'action politique. Dans le cas des croix de Malte à Saint-Christol ou dans celui du déversoir de Marsillargues, c'est un projet d'aménagement qui a focalisé l'action politique et le débat public. Pour les autres lieux étudiés, ce sont des pratiques spatiales quotidiennes qui ont été prises en compte et mises en lien avec les actions politiques et les débats publics. Ce changement d'échelle nous a alors permis de saisir un deuxième niveau de complexité du territoire en mettant en évidence une grande partie du «tissu d'événements, actions, interactions, rétroactions, déterminations, aléas, qui constituent notre monde phénoménal ${ }^{58}$ ».

Le territoire Lunellois est ainsi composé :

- d'événements : projet d'aménagement comme la construction d'un pôle œntouristique, inondations, marché de producteurs, etc.

- d'actions : actions politiques mais également actions des habitants à l'origine de leur pratiques spatiales (loisirs de plein air par exemple);

- d'interactions et de rétroactions entre actions politiques, débats publics et pratiques spatiales comme l'a montré l'ensemble du cas d'étude;

- de déterminations : les composantes physiques du territoire, qualité des sols qui influence les productions présentes, relief (exemple des falaises de la Roque-de-Saint-Sériès) ou encore proximité de l'agglomération montpelliéraine;

- d'aléas, c'est-à-dire d'éléments ponctuels non contrôlables : inondations, crise du marché viticole puis crise économique générale par exemple. 
Le prise en compte du plus petit échelon de complexité du territoire que nous avons dans cette étude appelé lieu agricole périurbain semble donc essentielle pour saisir plusieurs niveaux de complexité du territoire et pour dépasser l'objectivation initiale du chercheur. En effet, la prise en compte des pratiques spatiales, pourtant à l'origine de formes de constructions territoriales, ne peut se faire qu'à cette échelle. Dans cette perspective, nous pensons souhaitable que soit systématisé l'approfondissement des diagnostics de territoire par des zooms sur des lieux (agricoles ou autres). Cela permettrait de décrire précisément les pratiques spatiales à l'œuvre dans les territoires et de les relier aux actions politiques et débats publics en cours.

\section{Bibliographie}

Allemand, Rémi, Sylvain Rouanet et Jacques Gleyse, «La fête du taureau ou des taureaux dans la fête?», Sociétés, no 107, 2010, p. 73-86.

Angelini, Julien, "Le débat public comme agir territorial : méthodes qualitatives", Communication et organisation, $\mathrm{n}^{\circ} 38,2010$, p. 171-80.

Anthaume, Benoît et Frédéric Giraut, Le territoire est mort, vive les territoires! Une (re)fabrication au nom du développement, Paris, IRD Éditions, 2005.

Arendt, Hannah, La crise de la culture, Paris, Gallimard, 1989.

Boy, Daniel, "La place de la question environnementale dans le débat public", Regards croisés sur l'économie, n 6, 2009, p. 48-55.

Brennetot, Arnaud, «Des bases de données et du débat public dans les régions", L'espace géographique, tome 40, 2011, p. 133-37.

Chaze, Jean-Pierre, «La course camarguaise : un sport ou un jeu traditionnel? ", Corps et culture, $\mathrm{n}^{\circ} 4,1999$, p. 15.

Clément, Camille, La publicisation des espaces agricoles périurbains : du processus global à la fabrique du lieu, thèse de géographie, Université Montpellier 3, 2012.

Courcelle, Thibault, «Le rôle de la presse quotidienne régionale bretonne dans la création d'une "identité bretonne" : étude comparative de Ouest-France et du Télégramme», Hérodote, n 110, 2003, p. 129-148. 
Dahlgren, Peter, Marc Abélès, Daniel Dayan et Éric Maigret, «L'espace public et les médias : une nouvelle ère?», Hermès, $\mathrm{n}^{\text {os }} 13-14,1994$, p. 243-262.

Dear, Michael, «Understanding and Overcoming the NIMBY Syndrome ", Journal of the American Planning Association, vol. 58, $\mathrm{n}^{\circ}$ 3, 1992, p. 288-300.

Debarbieux, Bernard, "Le lieu, le territoire et trois figures de rhétorique», Espace géographique, vol. 24, n 2, 1995, p. 97-112.

Di Méo, Guy, Géographie sociale et territoires, Paris, Nathan Université, 2001.

Dodier, Rodolphe, Laurent Cailly, Arnaud Gasnier, et François Madoré, Habiter les espaces périurbains, Rennes, Presses universitaires de Rennes, 2012.

Foucault, Michel, Naissance de la biopolitique. Cours au collège de France, Paris, Presses universitaires de France, 2004.

Foucault, Michel, Surveiller et punir. Naissance de la prison, Paris, Gallimard, 1975.

Frémont, Armand, La région, espace vécu, Paris, Presses universitaires de France, 1976.

Gervais-Lambony, Philippe, Territoires citadins : 4 villes africaines, Paris, Belin, 2003.

Giraut, Frédéric, "Conceptualiser le territoire», Historiens et géographes, ${ }^{\circ}$ 403, 2008, p. 57-68.

Guérin-Pace, France, "Vers une typologie des territoires urbains de proximité", L'espace géographique, no 32, 2003, p. 333-344.

Gumuchian, Hervé et al., Les acteurs, ces oubliés du territoire, Paris, Economica, 2003.

Gumuchian, Hervé et Bernard Pecqueur, La ressource territoriale, Paris, Economica, 2007.

Institut National de la Statistique et des Études Économiques (INSEE) (2011), Statistiques sur l'EPCI de la CC du Pays de Lunel, 2011, [en ligne] http://www.insee.fr/fr/themes/dossier_complet.asp?codgeo=EPCI243400520, consulté en septembre 2014.

Lascoumes, Pierre et Patrick Le Galès, Gouverner par les instruments, Paris, Les Presses de Sciences Po, 2005.

Lay, Yves-François et Anne Rivière-Honegger, «Expliquer l'inondation : la presse quotidienne régionale dans les Alpes et leur piedmont (1882-2005)", Géocarrefour, vol. 84, n 4, 2009, p. 259-270.

Lazzarotti, Olivier, Les loisirs à la conquête des espaces périurbains, Paris, L'Harmattan, 2000. 
Le Caro, Yvon, Les loisirs en espace agricole. L'expérience d'un espace partagé, Rennes, Presses universitaires de Rennes, 2007.

Lévy, Jacques et Michel Lussault, Dictionnaire de la géographie et de l'espace des sociétés, Paris, Belin, 2003.

Lussault, Michel, L'Homme spatial. La construction sociale de l'espace humain, Paris, Seuil, 2007.

Moine, Alexandre, "Le territoire comme un système complexe : un concept opératoire pour l'aménagement et la géographie», L’espace géographique, $\mathrm{n}^{\circ} 35,2006$, p. 115-132.

Monnoyer-Smith, Laurence, «Le débat public en ligne : une ouverture des espaces et des acteurs de la délibération?", dans Martine Revel et al., Le débat public : une expérience française de démocratie participative, Recherches, 2007, p. 155-166.

Morin, Edgar, Introduction à la pensée complexe, Paris, ESF, 1990.

Pissaloux, Jean-Luc, "La démocratie participative dans le domaine environnemental ", Revue française d'administration publique, ${ }^{\text {os }} 137-138$, 2011, p. 123-136.

Raynaud, Claude, "Histoire d'un nectar : le muscat de Lunel», Études héraultaises, hors-série, 2011, p. 31-47.

Recensement Général Agricole, 2010, [en ligne] http://www.agreste. agriculture.gouv.fr/recensement-agricole-2010/.

Schéma de Cohérence Territoriale (SCoT) du Pays de Lunel, Communauté de communes du Pays de Lunel, 2006, p. 76.

Sintomer, Yves et Marie-Hélène Bacqué, La démocratie participative. Histoire et généalogie, Paris, La Découverte, 2011.

Soulard, Christophe Toussaint, Les agriculteurs et la pollution des eaux. Proposition d'une géographie des pratiques, thèse de géographie, Université Paris 1 La Sorbonne, 1999.

Subra, Philippe, "À quoi et à qui sert le débat public?», Hérodote, $\mathrm{n}^{\circ} 110$, 2003, p. 149-155.

Subra, Philippe, "Ce que le débat public nous dit du territoire et de son aménagement", Géocarrefour, nº 81, 2006, p. 287-298.

Torre, André et Christine Lefranc, «Les conflits dans les zones rurales et périurbaines. Premières analyses de la presse quotidienne régionale», Espaces et sociétés, $\mathrm{n}^{\text {os }} 124-125,2006$, p. 93-110. 